\title{
Cosmological constraints from clustering properties of galaxy clusters
}

\author{
A. Del Popolo ${ }^{1,2}$, N. Ercan $^{1}$, and I. S. Yeşilyurt ${ }^{1}$ \\ 1 Boğaziçi University, Physics Department, 80815 Bebek, Istanbul, Turkey \\ e-mail: antonino.delpopolo@boun.edu.tr \\ 2 Dipartimento di Matematica, Università Statale di Bergamo, via dei Caniana, 2, 24127, Bergamo, Italy
}

Received 25 June 2004 / Accepted 19 October 2004

\begin{abstract}
In this paper, we discuss improvements of the Suto et al. (2000) model, in the light of recent theoretical developments (new theoretical mass functions, a more accurate mass-temperature relation and an improved bias model) to predict the clustering properties of galaxy clusters and to obtain constraints on cosmological parameters. We re-derive the two-point correlation function of clusters of galaxies for OCDM and $\Lambda$ CDM cosmological models, and we compare these results with the observed spatial correlation function for clusters in RASS1 (ROSAT All-Sky Survey 1), and in XBACs (X-RAY Brighest Abell-Type) samples. The comparison shows that the best agreement is obtained for the $\Lambda$ CDM model with $\Omega_{\mathrm{m}}=0.3$. The values of the correlation length obtained, $\left(r_{0} \simeq 28.2 \pm 5.2 h^{-1} \mathrm{Mpc}\right.$ for $\left.\Lambda \mathrm{CDM}\right)$, are larger than those found in the literature and comparable with the results found in Borgani et al. (1999). In order to study the possible dependence of the clustering properties of the $\mathrm{X}$-ray clusters on the observational characteristics defining the survey, we calculated the values of the correlation length $r_{0}$ in the catalogues where we vary the limiting X-ray flux $S_{\text {lim. }}$. The result shows an increase of $r_{0}$ with $L_{\mathrm{lim}}$, and correlation lengths that are larger than in previous papers in literature (e.g. Moscardini et al. 2001 (hereafter MMM); Suto et al. 2000). These differences are due essentially to the different $M-T$, mass function and bias model used in this paper. Then, we perform a maximum-likelihood analysis by comparing the theoretical predictions to a set of observational data in the X-ray band (RASS1 Bright Sample, BCS (Rosat Brightest Cluster Sample), XBACs, REFLEX (ROSAT-ESO Flux Limited X-Ray Sample)), similarly to MMM. In the framework of cold dark matter models, we compute the constraints on cosmological parameters, such as the matter density $\Omega_{\mathrm{m}}$, the contribution to density due to the cosmological constant, $\Omega_{\Lambda}$, the power-spectrum shape parameter $\Gamma$ and normalization $\sigma_{8}$. If we fix $\Gamma$ and $\sigma_{8}$, at the values suggested by different observational datasets, we obtain (for flat cosmological models with varying cosmological constant $\Omega_{0 \Lambda}=1-\Omega_{0 \mathrm{~m}}$ ) constraints on the matter density parameter: $0.25 \leq \Omega_{0 \mathrm{~m}} \leq 0.45$ and $0.23 \leq \Omega_{0 \mathrm{~m}} \leq 0.52$ at the 95.4 and 99.73 per cent levels, respectively, which is $20-30 \%$ larger than the values obtained MMM. Leaving $\Gamma$, and $\Omega_{\mathrm{m} 0}$, free for the flat model, the constraints for $\Gamma$ are $0.1 \leq \Gamma \leq 0.14$, while for the open model $0.09 \leq \Gamma \leq 0.13$. These values are smaller than those of MMM by about $20-30 \%$. If we keep the values of $\Omega_{\Lambda}$ fixed, we obtain the constraints in the $\Gamma-\sigma_{8}$ plane. For the open model with $\Omega_{0 \mathrm{~m}}=0.3$ the $2 \sigma$ region for $\Gamma$ is $0.11-0.2$ for $\sigma_{8}$ it is 0.7 and 1.55. For the flat model with $\Omega_{0 \mathrm{~m}}=0.3$ the $2 \sigma$ region has $0.13 \leq \Gamma \leq 0.2$ and $0.8 \leq \sigma_{8} \leq 1.6$ The values of $\sigma_{8}$ obtained are larger than those of $\mathrm{MMM}$ by $\simeq 20 \%$. If we allow the shape parameter to vary, we find that the clustering properties of clusters are almost independent of the matter density parameter and of the presence of a cosmological constant, while they appear to be strongly dependent on the shape parameter.
\end{abstract}

Key words. cosmology: large-scale structure of Universe

\section{Introduction}

$\mathrm{X}$-ray studies of clusters of galaxies have provided a large amount of quantitative data for the study of cosmology. The mass of a rich cluster is approximately $10^{15} h^{-1} M_{\odot}{ }^{1}$. This mass lies within a region of diameter $\simeq 20 h^{-1} \mathrm{Mpc}$ and consequently the observations of clusters can provide information on the mass distribution of the Universe on these scales. Furthermore, since rich clusters are rare objects, their properties are expected

${ }^{1} h=H_{0} /\left(100 \mathrm{~km} \mathrm{~s}^{-1} \mathrm{Mpc}^{-1}\right), H_{0}$ being the Hubble constant at the current epoch (in the paper we adopt $h=0.65$ ) (see Spergel et al. 2003; Tegmark et al. 2004). to be sensitive to the underlying mass density field from which they originated. Therefore, clusters of galaxies appear to be ideal tools for testing theories of structure formation as well as studying large-scale structure.

$\mathrm{X}$-ray catalogues of X-ray selected clusters are now available from ROSAT: RASS1 (De Grandi et al. 1999) (ROSAT All-Sky survey 1), BCS (Ebeling et al. 1998) (ROSAT Brightest Cluster Sample), XBACs (Ebeling et al. 1996) (X-ray brightest Abell Cluster Sample), REFLEX (Böhringer et al. 1998) (ROSAT-ESO Flux-Limited X-ray sample) and the volume covered by the samples is expected to increase through the $\mathrm{X}$-ray satellites such as Astro-E, Chandra, and XMM. These 
data together with optical data have been used to compute the cluster number counts and the X-ray luminosity function, which have relevant cosmological implications. In particular, the analysis of the cluster abundance (also as a function of redshift) has been used widely to provide estimates of the mass fluctuation amplitude and of the matter density parameter $\Omega_{\mathrm{m}}$, with several, often discrepant results (Kitayama \& Suto 1997; Mathiesen \& Evrard 1998; Sadat et al. 1998; Reichart et al. 1999a,b; Viana \& Liddle 1999; Blanchard et al. 1998; Eke et al. 1998; Bahcall et al. 1997; Fan et al. 1997; Bahcall \& Fan 1998; Donahue \& Voit 1999; Borgani et al. 2001). An alternative approach to the abundance of clusters is based on the study of the spatial distribution of selected clusters. The standard statistical tools used with this aim are the (spatial and angular) two-point correlation function and the power-spectrum.

The two-point correlation function is a fundamental statistical test for the study of the cluster distribution and is relatively straightforward to determine from observational data. The spatial correlation function of galaxy clusters provides an important cosmological test, as both the amplitude of the correlation function and its dependence upon the mean intercluster separation are determined by the underlying cosmological model. Like for the cluster abundance, discrepant results have been found for, e.g., the correlation length (Hauser \& Peebles 1973; Bahcall \& Soneira 1983; Klypin \& Kopylov 1983; Bahcall \& Cen 1992; Bahcall \& West 1992; Efstathiou et al. 1992; Governato et al. 1999).

As shown in some papers (Eke et al. 1998; Reichart et al. 1999a,b; Donahue \& Voit 1999; Borgani et al. 2001; Del Popolo 2003), the reasons leading to the quoted discrepancies are not only connected to the observational data used, but other unknown systematic effects may be plaguing a large part of the quoted results (Reichart et al. 1999a,b; Eke et al. 1998; Donahue \& Voit 1999; Borgani et al. 2001). Systematic effects entering the quoted analyses are: 1) The inadequate approximation given by the Press-Schechter relation (e.g., Bryan \& Norman 1998). 2) Inadequacy in the structure formation as described by the spherical model leading to changes in the threshold parameter $\delta_{\text {c }}$ (e.g., Governato et al. 1999). 3) Inadequacy in the $M-T$ relation obtained from the virial theorem (see Voit \& Donahue 1998; Del Popolo 2002a). 4) Effects of cooling flows. 5) Missing high redshift clusters in the data used (e.g., the EMSS). 6) Evolution of the L-T relation.

Although the quoted uncertainties have so far been of minor importance with respect to the paucity of observational data, a breakthrough is needed in the quality of the theoretical framework if high-redshift clusters are to contribute to in the high-precision-era of observational cosmology.

Moreover, the proper comparison of the two-point correlation function with X-ray data, requires better theoretical predictions which take account of the selection function of X-ray clusters (Kitayama et al. 1998), the luminosity-and time dependent bias (Mo \& White 1996; Jing 1998; Moscardini et al. 1998), the light-cone effect (Matarrese et al. 1997; Matsubara et al. 1997; Nakamura et al. 1998; Yamamoto \& Suto 1999) and the redshift-space distortion (Hamilton 1998; Matsubara \& Suto 1996; Suto et al. 2000; Nishioka \& Yamamoto 1999; Yamamoto et al. 1999; Magira et al. 2000).
The above discussion and recent developments in terms of both theory (improved relations for the mass function, $M-T$ relation, and bias) and observation (X-ray data) suggest that it would be useful to recalculate the two-point correlation function and to revisit the constraints on cosmological parameters obtained until now.

Likely in Del Popolo (2003), in the present paper we are principally interested in studying the effects of these changes on the values of the cosmological parameters and in comparing them with previous estimates, and then in the specific values obtained. For this reason, we made a comparison of the theoretical results with observations using several samples such as RASS1 and XBAC, even if it is known that the REFLEX is more precise (small errorbars). The paper is organized as follows: in Sect. 2, we introduce the model used. Section 3 is devoted to the results and Sect. 4 to discussion and conclusions.

\section{Theoretical model}

\subsection{Redshift-space distortion}

In order to obtain a theoretical model for the spatial two-point correlation function of galaxies in different cosmologies, we follow and improve the paper of Suto et al. (2000, hereafter S2000). Their model takes proper account of nonlinear gravitational evolution of mass fluctuations, redshift-space distortion due to the linear peculiar velocity field and to finger-of-god effect, cluster abundance and bias evolution on the basis of the Press - Schechter theory, and the light-cone effect.

As previously reported, one of the effects to take into account is the two-point correlation function distortions due to the peculiar velocity field. We take into account this redshift-space distortion following Cole et al. (1994), Magira et al. (2000) and Yamamoto et al. (1999). Assuming that the bias of the cluster density field relative to the mass density field is linear and scale-independent, the power spectrum in redshift space is well approximated by:

$P_{\mathrm{cl}}^{\mathrm{S}}(k, \mu, z)=P_{\mathrm{mass}}^{\mathrm{R}}(k, z)\left[b_{\mathrm{cl}}(z)\right]^{2}\left[\frac{1+\beta(z) \mu^{2}}{1+\left(k \mu \sigma_{v}\right)^{2} / 2}\right]^{2}$,

where $P_{\text {mass }}^{\mathrm{R}}(k, z)$ is the mass power spectrum in real space, $\mu$ the direction cosine of the wavenumber vector and the lineof-sight of the fiducial observer, and $\beta$ is linear redshift-space distortion (Kaiser 1987), defined by

$\beta(z)=\frac{1}{b_{\mathrm{cl}}(z)} \frac{\mathrm{d} \ln D_{1}(z)}{\mathrm{d} \ln a(z)}$

where $b_{\mathrm{cl}}(z)$ is the redshift-dependent bias factor and $D_{1}(z)$ is the linear growth factor normalized to be unity at the present time.

The denominator in Eq. (1) takes account of the nonlinear redshift-space distortion (finger-of-God) assuming that the pair-wise velocity distribution in real space is exponential with the velocity dispersion of $\sigma_{\mathrm{v}}(z)$.

As in S2000, to calculate $\sigma_{\mathrm{v}}$ we use the fitting formula of Mo et al. (1997). Averaging Eq. (1) over the angle with respect to the line-of-sight of the observer one obtains $P_{\mathrm{cl}}^{\mathrm{S}}(k, z)$ 
similarly to S2000 (Eqs. (4)-(7)). The corresponding two-point correlation function of clusters in redshift space is computed as

$\xi_{\mathrm{cl}}^{\mathrm{S}}(R, z)=\frac{1}{2 \pi^{2}} \int_{0}^{\infty} \mathrm{d} k k^{2} P_{\mathrm{cl}}^{\mathrm{S}}(k, z) j_{0}(k R)$,

where $j_{0}(k R)$ is the spherical Bessel function.

\subsection{The evolution of the mass auto-correlation function}

To predict the clustering properties of X-ray clusters, we need a description of the matter covariance function and its redshift evolution. To this aim we used the method of Smith et al. (2003), which is an improvement of the method of Peacock \& Dodds (1994), and Peacock \& Dodds $(1996)^{2}$ for evolving $\xi(r, z)$ into the fully non-linear regime. The authors adopted a new approach to fitting power spectra, based upon a fusion of the halo model and HKLM (Hamilton, Kumar, Lu, Matthews) scaling. This approach has been empirically shown to allow an accurate description of a very wide range of power spectrum data. Their formula reproduced the scale-free power spectrum data and also the CDM results of Jing (1998) with an rms error better than 7\% (see Smith et al. (2003) for more details and their Appendix for the fitting formula).

The linear power spectrum used in this paper, $P_{\mathrm{L}} \propto$ $k^{n} T^{2}(k)$, uses the Bardeen et al. (1986) transfer function $T(k)$ (Bardeen et al. 1986, Eq. (G3)), and the shape parameter $\Gamma$ is given by:

$\Gamma=\Omega_{0 \mathrm{~m}} h \exp \left(-\Omega_{0 \mathrm{~b}}-\sqrt{h / 0.5} \Omega_{0 \mathrm{~b}} / \Omega_{0 \mathrm{~m}}\right)$,

(Sugiyama 1995), where $\Omega_{0 \mathrm{~m}}$ is the baryonic contribution to the density parameter. In the part of the paper dealing with the direct comparison with XBACs and RASS1 data, we consider an open CDM model (OCDM), with matter density parameter $\Omega_{0 \mathrm{~m}}=0.3$ and $\sigma_{8}=0.87$, and a low-density flat CDM model $\left(\Lambda \mathrm{CDM}\right.$ ), with $\Omega_{0 \mathrm{~m}}=0.3$, and $\sigma_{8}=0.93$ (see e.g. Liddle et al. $1996 \mathrm{a}, \mathrm{b}$ and references therein).

In the part of the paper dealing with the maximumlikelihood analysis the value of $\Gamma$ is allowed to vary in the range $0.05-0.5$, while $\Omega_{0 \mathrm{~m}}$ ranges from 0.1 to 1 in the framework of both open and flat models. The normalizations of the primordial power-spectrum, parameterized by $\sigma_{8}$ (the rms fluctuation amplitude in a sphere of $8 \mathrm{~h}^{-1} \mathrm{Mpc}$ ) is allowed to vary in the range $0.2 \leq \sigma_{8} \leq 2$. In the maximum-likelihood analysis the cosmological models considered, are defined by four parameters: $\Omega_{0 \mathrm{~m}}, \Omega_{0 \Lambda}, \Gamma$ and $\sigma_{8}$.

\subsection{Bias evolution}

In order to predict the clustering properties of clusters as a function of redshift, we need to know how bias evolves. S2000 adopted for the "monochromatic" bias $b(M, z)$ the expression which holds for virialized dark matter haloes (see their Eq. (17)) (e.g. Mo \& White 1996; Catelan et al. 1998), and to get the effective bias factor the Mo \& White (1996) equation

\footnotetext{
${ }^{2}$ Based on Hamilton et al. (1991) original ansatz.
}

was combined with the Press-Schechter relation to translate the quoted bias factor into a function of X-ray flux limit (see their Eq. (18)).

Several papers in literature has shown that the Mo \& White (1996) bias formula does not correctly reproduce the correlation of low mass haloes in numerical simulations.

Several alternative fits have been proposed (Del Popolo \& Gambera 1998; Jing 1998; Porciani et al. 1999; Sheth \& Tormen 1999; Jing 1999; Del Popolo 2001). The bias model of Sheth \& Tormen (1999) has been shown to produce an accurate fit of the distribution of the halo populations in the GIF simulations (Kauffmann et al. 1999).

In this paper we adopt the bias model described in Del Popolo \& Gambera (1998), and Del Popolo (2001), because it produces a mass function that is in better agreement with the predictions of Jenkins et al. (2001) (see below). The quoted biased model is based on the threshold:

$\delta_{\mathrm{c}}=\delta_{\mathrm{co}}\left[1+\int_{r_{\mathrm{i}}}^{r_{\mathrm{ta}}} \frac{r_{\mathrm{ta}} L^{2} \cdot \mathrm{d} r}{G M^{3} r^{3}}\right]$

where $\delta_{\mathrm{co}}=1.68$ is the critical threshold for a spherical model, $r_{\mathrm{i}}$ is the initial radius, $r_{\mathrm{ta}}$ is the turn-around radius, and $L$ is the angular momentum ${ }^{3}$. Equation (5), is obtained taking account of the effect of asphericities. In Fig. 1, we plot the bias parameter, $b$, as a function of the peak height $v^{4}$, which is proportional to the halo mass. The solid line shows the spherical collapse prediction of Mo \& White (1996), the dotted line the prediction for $b$ obtained from our model, and the dashed line the ellipsoidal collapse prediction of Sheth \& Tormen (1999). As shown in the figure, taking account of the effects of asphericity produces a change in the dependence of $b$ on $v$ in good agreement with Sheth \& Tormen (1999). From Fig. 1 it is evident that at the low mass end the bias relation has an upturn, meaning that less massive haloes are more strongly clustered than in the prediction of the spherical collapse model of Mo \& White (1996) and in agreement with $N$-body simulations (Jing 1998; Sheth \& Lemson 1999; Kauffmann et al. 1999).

The above bias factor can be translated into a function of $\mathrm{X}$-ray flux limit according to:

$b_{\text {eff }}\left(z,>S_{\lim }\right)=\frac{\int_{M_{\lim }\left(S_{\lim }\right)}^{\infty} \mathrm{d} M b(z, M) n(z, M)}{\int_{M_{\lim }}^{\infty} \mathrm{d} M n(z, M)}$

where $n(z, M)$ is the number of objects actually present in the catalogue with redshift in the range $z, z+\mathrm{d} z$ and $M$ in the range $M, M+\mathrm{d} M$. One can estimate $n(z, M)$ from the Press-Schechter (1974, PS) formula; however, several studies have shown some discrepancies between PS and simulations.

\footnotetext{
${ }^{3}$ The angular momentum appearing in Eq. (5) is the total angular momentum acquired by the proto-structure during evolution. In order to calculate $L$, We will use the same model as described in Del Popolo \& Gambera $(1998,1999)$ (more hints on the model and some of the model limits can be found in Del Popolo et al. 2001).

${ }^{4} v=\left(\frac{\delta_{c}(z)}{\sigma(M)}\right)^{2}$ is the ratio between the critical overdensity required for collapse in the spherical model, $\delta_{\mathrm{c}}(z)$, to the rms density fluctuation $\sigma(M)$, on the scale $r$ of the initial size of the object $M$.
} 


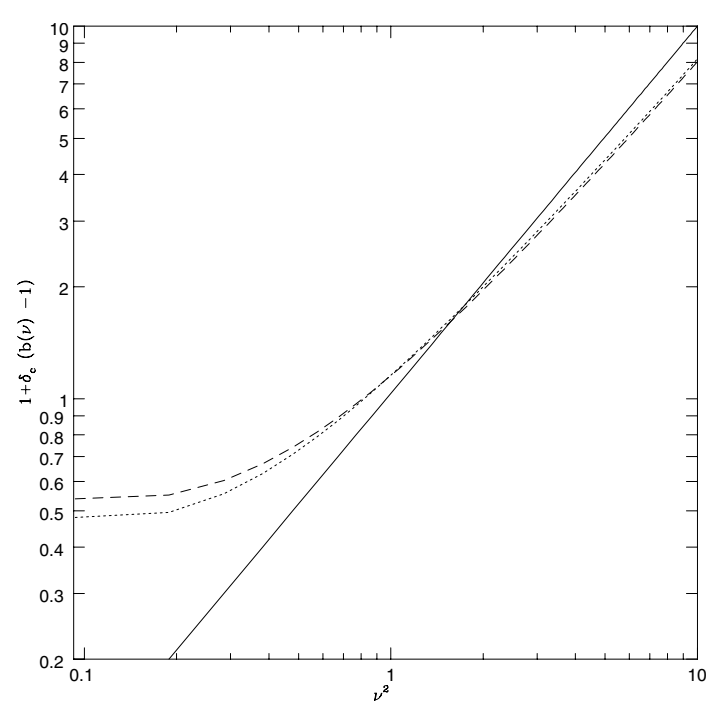

Fig. 1. The bias factor $b(v)$ as a function of $v^{2}$. The solid line represents the spherical collapse prediction of Mo \& White (1996), the dotted line the prediction for $b$ obtained from the model of this paper and the dashed line the ellipsoidal collapse prediction of Sheth \& Tormen (1999).

In order to obtain a better estimate of $n(M, z)$, we can use the excursion set approach which allows one to calculate good approximations to several important quantities, such as the "unconditional" and "conditional" mass functions. Sheth \& Tormen (2002, hereafter ST) provided formulas to calculate these last quantities starting from the shape of the barrier. They also showed that the "unconditional" mass function, which is the one we need now, is in good agreement with results from numerical simulations. Using the barrier shape obtained in Del Popolo \& Gambera (1998), obtained from the parameterization of the nonlinear collapse discussed in that paper, together with the results of ST we can calculate the "unconditional" mass function.

Assuming that the barrier is proportional to the threshold for the collapse, as in ST, the barrier can be expressed in the form:

$B(M)=\delta_{\mathrm{c}}=\delta_{\mathrm{co}}\left[1+\int_{r_{\mathrm{i}}}^{r_{\mathrm{ta}}} \frac{r_{\mathrm{ta}} L^{2} \cdot \mathrm{d} r}{G M^{3} r^{3}}\right] \simeq \delta_{\mathrm{co}}\left[1+\frac{\beta_{1}}{v^{\alpha_{1}}}\right]$

(Del Popolo 2001), where $\delta_{\text {co }}=1.68$ is the critical threshold for a spherical model, $r_{\mathrm{i}}$ is the initial radius, $r_{\mathrm{ta}}$ is the turn-around radius, $L$ the angular momentum, $\alpha_{1}=0.585$ and $\beta_{1}=0.46$.

As described in Del Popolo (2002b), the mass function can be approximated by:

$$
\begin{aligned}
n(M, z)= & \frac{\bar{\rho}}{M^{2}} \frac{\mathrm{d} \log v}{\operatorname{dlog} M} v f(v) \simeq 1.21 \frac{\bar{\rho}}{M^{2}} \frac{\mathrm{d} \log (v)}{\operatorname{dlog} M} \\
& \times\left(1+\frac{0.06}{(a v)^{0.585}}\right) \sqrt{\frac{a v}{2 \pi}} \\
& \times \exp \left\{-a v\left[1+\frac{0.57}{(a v)^{0.585}}\right]^{2} / 2\right\}
\end{aligned}
$$

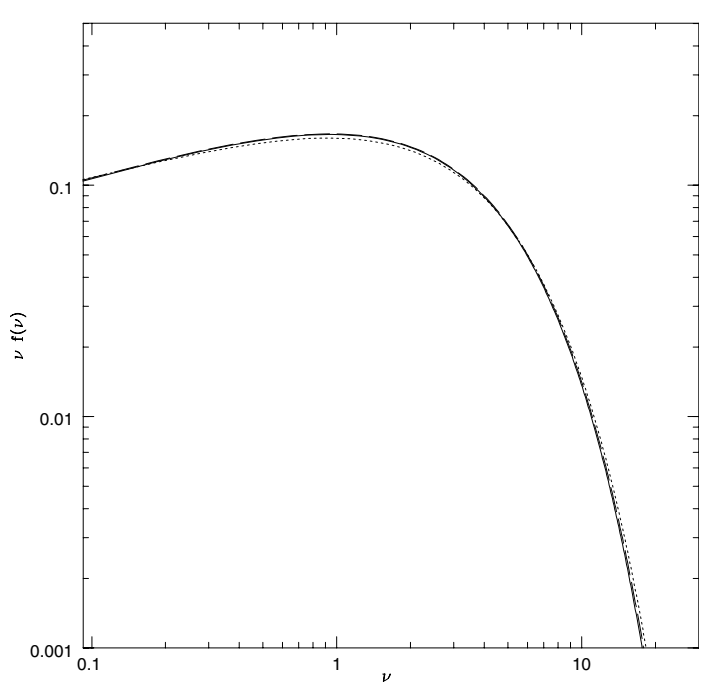

Fig. 2. Comparison of various mass functions. The dotted line represents the Sheth \& Tormen (2002) prediction, the solid line that of Jenkins et al. (2001) and the dashed line that of Del Popolo (2002b).

where $a=0.707$, and $\bar{\rho}$ is the background density ${ }^{5}$. In Fig. 2 we plot a comparison of the various mass functions: the dotted line represents Sheth \& Tormen (2002) prediction, the solid line that of Jenkins et al. (2001) and the dashed line that of Del Popolo (2002b). As Fig. 2 shows, the mass function obtained in this paper is in very good agreement with that of Jenkins et al. (2001) in the regime probed by the simulations. Notice that a large part of the constraints obtained in the past has been obtained using the PS mass function. Only in some more recent papers has the mass function been calculated by means of ST model (e.g. Borgani et al. 2001) or that of Jenkins et al. (2001) (Hamana et al. 2001; MMM). Moreover the $M-T$ relation chosen is the usual one obtained simply from the virial theorem (see next subsection). In other words, this paper introduces noteworthy improvements on the previous calculations in literature.

Before concluding this subsection, following Hamana et al. (2001), we further attempt to incorporate the scale-dependence on the basis of the results of Taruya \& Suto (2000), in which the scale-dependence arises as a natural consequence of the formation epoch distribution of halos. Yoshikawa et al. (2001), had shown that the scale-dependence of the Taruya \& Suto (2000) model agrees with their numerical simulations. Therefore we construct an empirical halo bias model of the two-point statistics which reproduces the scale-dependence of the Taruya \& Suto (2000) bias with the amplitude fixed by the massdependent bias obtained in Del Popolo \& Gambera (1998) and Del Popolo (2001). Following Hamana et al. (2001), the scale dependent bias shall be described by the following simple fitting formula:

$b_{\mathrm{h}}(M, R, z)=b(M, z)\left[1.0+b(M, z) \sigma_{R}(R, z)\right]^{0.15}$,

for $R>2 R_{\mathrm{vir}}(M, z)$, and otherwise 0 , where $R_{\mathrm{vir}}(M, z)$ is the virial radius of the halo of mass $M$ at $z$ and $\sigma_{R}(R, z)$ is the mass variance smoothed over the top-hat radius $R$. The bias factor $b$

\footnotetext{
${ }^{5}$ Note that in this formula $v=\left(\frac{\delta_{\mathrm{c}}(z)}{\sigma(M)}\right)^{2}$.
} 


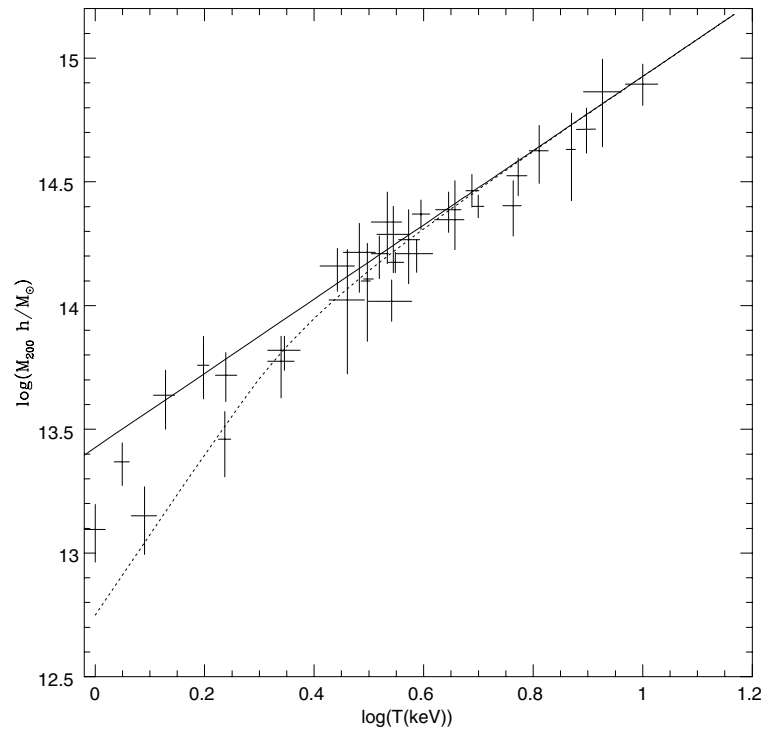

(a)

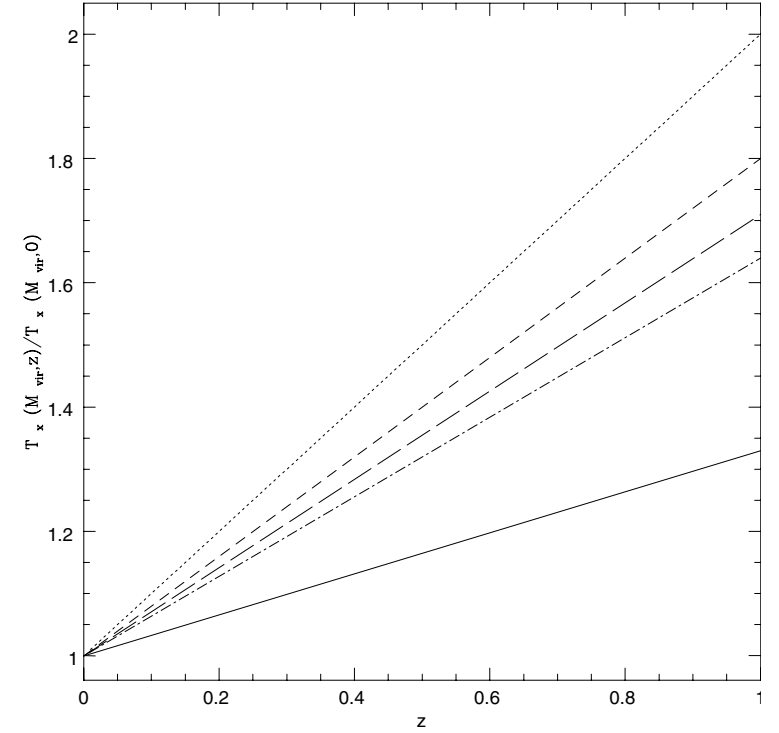

(b)

Fig. 3. a) $M-T$ relation predicted by the modified continous cluster formation model. The solid line is the prediction of the quoted model, described in Del Popolo (2002a), for $\Omega_{\Lambda}=0, \Omega_{0}=1$, shifted downwards, similarly as in Afshordi \& Cen (2002), to fit the Finoguenov et al. (2001) observational data at the massive end. The dotted line represents the prediction of the same model for $\Omega_{\Lambda}=0.7$ and $\Omega_{0}=0.3$. b) Temperature evolution predicted by the modified continous cluster formation model in the case $\Omega_{0}=0.3$. The dotted line represents the "classical" prediction, $T_{\mathrm{X}} \propto(1+z)$. The short-dashed line represents the late-formation approximation as expressed by Eq. (8) in Voit (2000), namely $T_{\mathrm{X}} \propto \Delta_{\text {vir }}^{1 / 3}\left[\frac{\Omega_{0}}{\Omega_{0}(z)}\right]^{1 / 3}(1+z)$. The long-dashed line and the dot-dashed one plot Eq. (40) in Del Popolo (2002a) for the spherical collapse model $n=-2, n=-1$, respectively. The solid line plots the same equation for $n=-1$ and taking account of angular momentum acquisition by the protostructure.

contained in Eq. (3) through the spectrum $P_{\mathrm{cl}}^{\mathrm{S}}$ should then be substituted by Eq. (9).

Although the modeling is not completely self-consistent in the sense that the scale-dependence of the halo biasing factor is neglected in describing the redshift distortion, the above prescription provides a good approximation since the scaledependence in the biasing is of secondary importance in the redshift distortion effect of halos (see Hamana et al. 2001).

\subsection{Limiting flux and halo mass}

In order to predict the abundance and clustering of X-ray clusters in a given sample, it is necessary to relate the $\mathrm{X}$-ray fluxes to the corresponding halo mass at each redshift. As a first step, we relate the total mass of the dark halo of a cluster to the temperature of the gas. The $M-T$ relation that we shall use is that calculated in Del Popolo (2002a). The $M-T$ relation is calculated using the merging-halo formalism of Lacey \& Cole (1993), which takes account of the fact that massive clusters accrete matter quasi-continuously, and the present paper is an improvement of a model proposed by Voit (2000) (hereafter V2000), again to take account of angular momentum acquisition by protostructures and of an external pressure term in the virial theorem. The $M-T$ relation obtained in Del Popolo (2002a), is given by:

$k T \simeq 8 \mathrm{keV}\left(\frac{M^{\frac{2}{3}}}{10^{15} h^{-1} M_{\odot}}\right) \frac{\left[\frac{1}{m}+\left(\frac{t_{\Omega}}{t}\right)^{\frac{2}{3}}+\frac{K(m, x)}{M^{8 / 3}}\right]}{\left[\frac{1}{m}+\left(\frac{t_{\Omega}}{t_{0}}\right)^{\frac{2}{3}}+\frac{K_{0}(m, x)}{M_{0}^{8 / 3}}\right]}$, where $t_{\Omega}=\frac{\pi \Omega_{0 m}}{H_{0}\left(1-\Omega_{0 m}-\Omega_{0 \Lambda}\right)^{\frac{3}{2}}}, m=5 /(n+3)$ (where $n$ is the spectral index), $M_{0}$ is defined in Del Popolo (2002a), and:

$$
\begin{aligned}
K(m, x)= & F x(m-1) \operatorname{LerchPhi}(x, 1,3 m / 5+1) \\
& -F(m-1) \operatorname{LerchPhi}(x, 1,3 m / 5),
\end{aligned}
$$

where $F$ is defined in Del Popolo (2002a) (Eq. (35)) and the LerchPhi function is defined as follows:

$\operatorname{LerchPhi}(z, a, v)=\sum_{n=0}^{\infty} \frac{z^{n}}{(v+n)^{a}}$,

and where $K_{0}(m, x)$ indicates that $K(m, x)$ must be calculated assuming $t=t_{0}$.

Equation (10) takes account of the fact that massive clusters accrete matter quasi-continuously, and also of tidal interaction between clusters. The obtained $M-T$ relation is no longer selfsimilar, there is a break at the low mass end $(T \sim 3-4 \mathrm{keV})$ of the $M-T$ relation is present. The behavior of the $M-T$ relation is as usual, $M \propto T^{3 / 2}$, at the high mass end, and $M \propto T^{\gamma}$, with a value of $\gamma>3 / 2$ dependening on the chosen cosmology. Larger values of $\gamma$ are related to open cosmologies, while $\Lambda$ CDM cosmologies give a slope intermediate between the flat case and the open case.

In Fig. 3a, we plot the $M-T$ relation predicted by the modified continous formation model. The solid line is the prediction of the quoted model, described in Del Popolo (2002a), for $\Omega_{\Lambda}=0, \Omega_{0}=1$, shifted downwards, as in Afshordi \& Cen (2002), to fit the FRB observational data at the massive end. The dotted line represents the prediction of the same model for 
$\Omega_{\Lambda}=0.7$ and $\Omega_{0}=0.3$. In Fig. $3 \mathrm{~b}$, we plot the temperature evolution predicted by the modified model for $\Omega_{0}=0.3$. The dotted line represents the "classical" prediction, $T_{\mathrm{X}} \propto(1+z)$. The short-dashed line represents the late-formation approximation as expressed by Eq. (8) in Voit (2000), namely $T_{\mathrm{X}} \propto$ $\Delta_{\text {vir }}^{1 / 3}\left[\frac{\Omega_{0}}{\Omega_{0}(z)}\right]^{1 / 3}(1+z)$. The long-dashed line and the dot-dashed one plot Eq. (40) in Del Popolo (2002a) for the spherical collapse model $n=-2, n=-1$, respectively. The solid line plots the same equation for $n=-1$ and taking account of angular momentum acquisition by the protostructure.

The next step (see S2000) is to transform the temperature to the luminosity of clusters using the observed luminositytemperature relation. In S2000, they assumed:

$L_{\mathrm{bol}}=L_{44}\left(\frac{T_{\mathrm{gas}}}{6 \mathrm{keV}}\right)^{\alpha}(1+z)^{\zeta} 10^{44} h^{-2} \mathrm{erg} \mathrm{s}^{-1}$

with $L_{44}=2.9, \alpha=3.4$ and $\zeta=0$ on the basis of the quoted observational indications (e.g., David et al. 1993; Ebeling et al. 1996; Ponman et al. 1996; Mushotzky \& Scharf 1997).

Several independent analyses of nearby clusters with $T_{\mathrm{X}} \geq$ $1 \mathrm{keV}$ consistently show that $L_{44} \simeq 3$ and $\alpha \simeq 2.5-3.5$ (e.g., White et al. 1997; Wu et al. 1999, and references therein). For cooler groups, $\leq 1 \mathrm{keV}$, the $L_{\mathrm{bol}}-T$ relation steepens. Mushotzky \& Scharf (1997) found that data out to $z \simeq 0.4$ are consistent with no redshift evolution in the $L_{\mathrm{bol}}-T$ relation out to $z \simeq 0.4$. In Moscardini et al. (2000a,b) the authors translated the cluster bolometric luminosity into a temperature, adopting the empirical relation

$T=\mathcal{A} L_{\mathrm{bol}}^{\mathcal{B}}(1+z)^{-\eta}$

where the temperature is expressed in $\mathrm{keV}$ and $L_{\mathrm{bol}}$ is in units of $10^{44} h^{-2} \mathrm{erg} / \mathrm{s}$ and $\mathcal{A}=4.2$ and $\mathcal{B}=1 / 3$, which are a good representation of the data with $T \geq 1 \mathrm{keV}$ (e.g. Markevitch 1998, and references therein).

From what was previously said, it is clear that the $L-T$ relation is a source of uncertainties. As in Del Popolo (2003), in the present paper we are principally interested in studying the effects of the improvements on the $M-T$ relation, mass function and bias model on cosmological parameters and to compare them with previous estimates. For this reason, in the following, we shall follow the philosophy of Moscardini et al. (2000a), Borgani et al. (1999), namely we shall adopt a "default" value for $\alpha$ and $L_{44},\left(L_{44}=2.9\right.$, and $\alpha=3.4$ as the reference values), and we calculate the correlation function and the constraints on cosmological parameters. We shall compare these with the results of previous papers (e.g., Moscardini et al. 2000a,b; S2000). Finally, we shall calculate the effects of the variation of $\alpha$ (in the range $2.5 \leq \alpha \leq 3.5$ ) on the resulting model constraints. Notice that all plots shown in the next sections are based on these "default" values $L_{44}=2.9$, and $\alpha=3.4$.

After fixing the $L-T$ relation, $L_{\text {bol }}\left(T_{\text {gas }}\right)$ is transformed in the band-limited luminosity $L_{\text {band }}\left[T_{\text {gas }}, E_{1}, E_{2}\right]$ as shown by S2000 (Sect. 2.2).

To obtain $M_{\text {lim }}$, necessary to calculate $b_{\text {eff }}$ in Eq. (6), we use the method of S2000 (see their Sect. 2.4)

\subsection{The light-cone effect}

The final step is to calculate the two-point correlation function on the light cone (Yamamoto \& Suto 1999):

$$
\xi_{\mathrm{X}-\mathrm{cl}}^{\mathrm{LC}}\left(R ;>S_{\lim }\right)=\frac{\int_{z_{\max }}^{z_{\min }} \mathrm{d} z \frac{\mathrm{d} V_{\mathrm{c}}}{\mathrm{d} z} n_{0}^{2}(z) \xi_{\mathrm{cl}}^{\mathrm{S}}\left(R, z(r) ;>S_{\lim }\right)}{\int_{z_{\max }}^{z_{\min }} \mathrm{d} z \frac{\mathrm{d} V_{\mathrm{c}}}{\mathrm{d} z} n_{0}^{2}(z)}
$$

where $R$ is the comoving separation of a pair of clusters, $z_{\max }$ and $z_{\mathrm{min}}$ denote the redshift range of the survey, and $\xi_{\mathrm{cl}}^{\mathrm{S}}(R, z ;>$ $\left.S_{\text {lim }}\right)$ is the corresponding two-point correlation function on a constant-time hypersurface at $z$ in redshift space (Eq. (3)). The comoving number density of clusters in the flux-limited survey, $n_{0}\left(z ;>S_{\text {lim }}\right)$, is computed by integrating the mass function Eq. (8) as:

$n_{0}\left(z ;>S_{\lim }\right)=\int_{M_{\lim }\left(S_{\lim }\right)}^{\infty} n(M, z) \mathrm{d} M$.

Finally the comoving volume element per unit solid angle is

$\frac{\mathrm{d} V_{\mathrm{c}}}{\mathrm{d} z}=\frac{d_{\mathrm{C}}^{2}(z)}{H(z)}$

where:

$H(z)=H_{0} \sqrt{\Omega_{0 \mathrm{~m}}(1+z)^{3}+\left(1-\Omega_{0 \mathrm{~m}}-\Omega_{0 \Lambda}\right)(1+z)^{2}+\Omega_{0 \Lambda}}$.

\subsection{Maximum-likelihood analysis}

In order to obtain constraints for cosmological models, we use a maximum-likelihood analysis. One possibility to accomplish the quoted analysis is as shown by Marshall et al. (1983), Del Popolo (2003), or Borgani et al. (1998).

In the present paper, We used the same model as MMM. The likelihood is $\mathcal{L} \propto \exp \left(-\chi^{2} / 2\right)$, where

$\chi^{2}=\sum_{i=1}^{N_{\text {data }}} \frac{\left[r_{0}(i)-r_{0}\left(i ; \Omega_{0 \mathrm{~m}}, \Omega_{0 \Lambda}, \Gamma, \sigma_{8}\right)\right]^{2}}{\sigma_{r_{0}}^{2}(i)}$.

The sum runs over the observational dataset described in Sect. 2 of MMM, i.e. $N_{\text {data }}=3$ and $N_{\text {data }}=4$ for the optical and X-ray bands, respectively (in the present paper we shall use only $\mathrm{X}$-ray data). The quantities $r_{0}(i)$ and $\sigma_{r_{0}}(i)$ represent the values of the correlation length and its $1 \sigma$ errorbar for each catalogue, as reported in Table 1 of MMM; $r_{0}\left(i ; \Omega_{0 \mathrm{~m}}, \Omega_{0 \Lambda}, \Gamma, \sigma_{8}\right)$ is the corresponding theoretical prediction obtained with a given choice of cosmological parameters. The best-fit cosmological parameters are obtained by maximizing $\mathcal{L}$, i.e. by minimizing $\chi^{2}$. The 95.4 and 99.73 per cent confidence levels for the parameters are computed by finding the region corresponding to an increase $\Delta_{\chi^{2}}$ with respect to the minimum value of $\chi^{2}$. Other details of the maximum-likelihood analysis are given in the next section. 


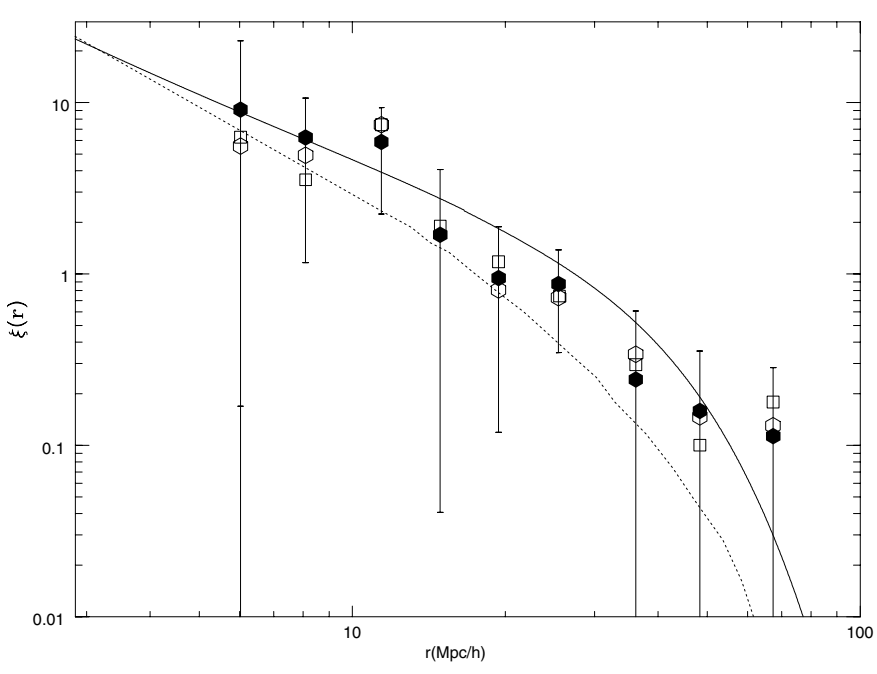

Fig. 4. Comparison of the cluster space correlations in the RASS 1 sample with the theoretical model of the present paper. In the plot, the dashed line represents the prediction of Moscardini et al. (2000b) for their $\Lambda \mathrm{CDM}$ model, and the solid line that of the $\Lambda \mathrm{CDM}\left(\Omega_{0 \mathrm{~m}}=0.3\right.$, $\sigma_{8}=0.93$ ) calculated using the model of this paper.

\section{Results}

We begin the analysis by comparing the theoretical predictions for the two-point correlation function to the observational clustering properties of RASS1 and afterwards to those of XBACs.

Figure 4 shows the comparison with RASS1. In the plot, the dashed line represents the prediction of Moscardini et al. (2000b) for their $\Lambda$ CDM model, and the solid line represents the prediction of the $\Lambda \mathrm{CDM}$, with $\Omega_{0 \mathrm{~m}}=0.3, \sigma_{8}=0.93$, and $\Gamma=0.21$ (see e.g. Liddle et al. 1996a,b and references therein), for the "default" value of $\alpha=3.4$. The plot for the OCDM model was not reported since it gives very similar predictions to the $\Lambda \mathrm{CDM}$ model. The $\Lambda \mathrm{CDM}$ model is in good agreement with the data and the predictions are always inside the $1 \sigma$ errorbars, $r_{0} \simeq 28.2 h^{-1} \mathrm{Mpc}$. A comparison with the Moscardini et al. (2000b) results, after the latter has been corrected taking account of the description of clustering in the past-light cone, (which gives $r_{0} \simeq 22.4 h^{-1} \mathrm{Mpc}$ ), shows that in our model the correlations are higher, by more than $20 \%$. Varying the value of $\alpha$ in the range 2.5-3.5, not plotted in Fig. 4, the resulting spatial correlation function does not change its shape, but only its amplitude: the smaller $\alpha$ is, the smaller $\xi(r)$ is. However, the changes are quite small, as shown in Fig. 11 of Moscardini et al. (2000a). The induced change of $r_{0}$, can be written as $r_{0} \simeq 28.2 \pm 5.2 \mathrm{~h}^{-1} \mathrm{Mpc}$.

In Fig. 5 the model is compared with the XBACs catalogue. Abadi et al. (1998) found that the XBACs spatial correlation function can be fitted by the usual power-law relation $\xi(r)=\left(r / r_{0}\right)^{-\gamma}$ with $\gamma=1.92$ and $r_{0}=21.1_{-2.3}^{+1.6} h^{-1} \mathrm{Mpc}$ (the errors correspond to $1 \sigma$ ), while Borgani et al. (1999), who adopted an analytical approximation to the bootstrap errors for the variance of $\xi(r)$, found $\gamma=1.98_{-0.53}^{+0.35}$ and a slightly larger value of $r_{0}=26.0_{-4.7}^{+4.1} h^{-1} \mathrm{Mpc}$ (the errors in this case are 2- $\sigma$ uncertainties). In Fig. 5, these observational estimates are compared with the theoretical predictions of this paper. The observational estimates are shown by two regions: the first one

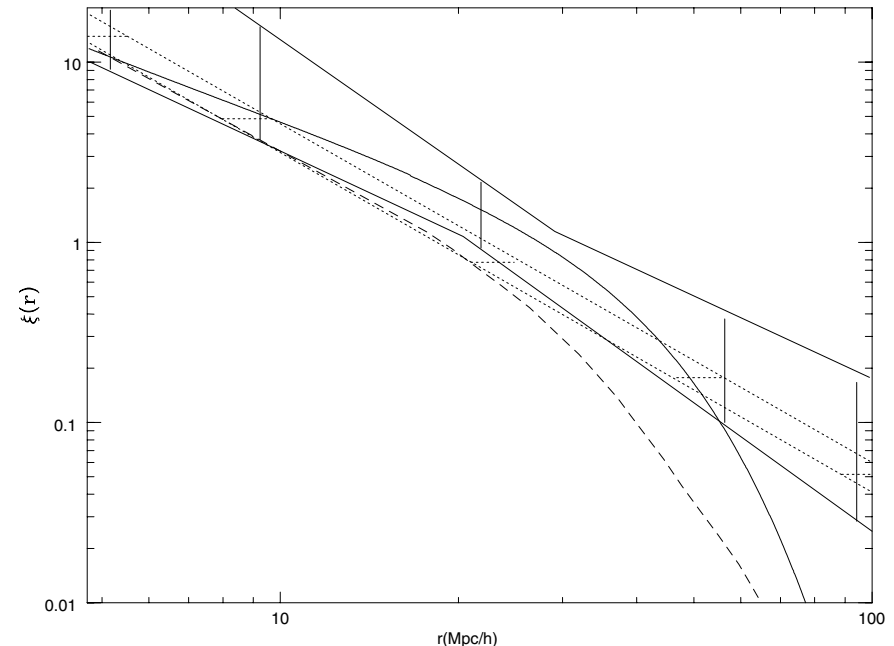

Fig. 5. Comparison of the cluster space correlations in the XBACs sample with the theoretical model of this paper. The observational estimates are shown by two regions: the first (enclosed in the solid lines connected by the vertical solid lines), refers to the $(1 \sigma)$ estimates obtained by Abadi et al. (1998), while the second region (enclosed in the dashed lines connected by the horizontal dashed lines), shows the $(2 \sigma)$ estimates by Borgani et al. (1999). The solid curve represents the $\Omega_{0 \mathrm{~m}}=0.3, \sigma_{8}=0.93 \Lambda \mathrm{CDM}$ model calculated using the model of this paper, while the dashed line represents the prediction of Moscardini et al. (2000a) for their $\Lambda \mathrm{CDM}$ model.

(enclosed in the solid lines connected by the vertical solid lines), refers to the $(1 \sigma)$ estimates obtained by Abadi et al. (1998), while the second region (enclosed in the dashed lines connected by the horizontal dashed lines), shows the $(2 \sigma)$ estimates by Borgani et al. (1999). The solid curve represents the $\Omega_{0 \mathrm{~m}}=0.3, \sigma_{8}=0.93 \Lambda \mathrm{CDM}$ model, while the dashed line represents the prediction of Moscardini et al. (2000a) for their $\Lambda \mathrm{CDM}$ model $^{6}$.

The results are in qualitative agreement with MMM, but the value for $r_{0}\left(r_{0} \simeq 28.2 \mathrm{~h}^{-1} \mathrm{Mpc}\right)$ obtained here is larger than the value in the quoted paper $\left(r_{0} \simeq 22 h^{-1} \mathrm{Mpc}\right.$ for $\left.\Lambda \mathrm{CDM}\right)$, while it is in better agreement with that of Borgani et al. (1999), who found a value of $r_{0}=26_{-4.7}^{+4.1} \mathrm{~h}^{-1} \mathrm{Mpc}$. Similar considerations to those relative to Fig. 4 are valid if we vary the value of $\alpha$.

In order to study the possible dependence of the clustering properties of the X-ray clusters on the observational characteristics defining the survey, we plot the values of the correlation length $r_{0}$ in the catalogues where we vary the limiting X-ray

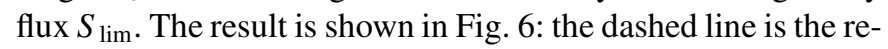
sult obtained by MMM, and the solid line that using the model of this paper, in the case of a $\Lambda \mathrm{CDM}$ model. The other cosmological models, not plotted, have a similar behavior but smaller amplitude (see MMM). In Fig. 6, $r_{0}$ increases with $L_{\mathrm{lim}}$. The increase is more rapid for $\Lambda \mathrm{CDM}$ models (see MMM). A similar analysis has been made by S2000 (see their Fig. 8) and MMM. Even if the S2000 results cannot be directly compared with those of MMM, (differences in cosmological parameters, formalism for the past-light cone effect, etc.) or even with those

\footnotetext{
${ }^{6}$ For the $\Lambda \mathrm{CDM}$ model (solid line), the results are in good agreement with the observational data $\left(r_{0} \simeq 28.2 h^{-1} \mathrm{Mpc}\right)$.
} 


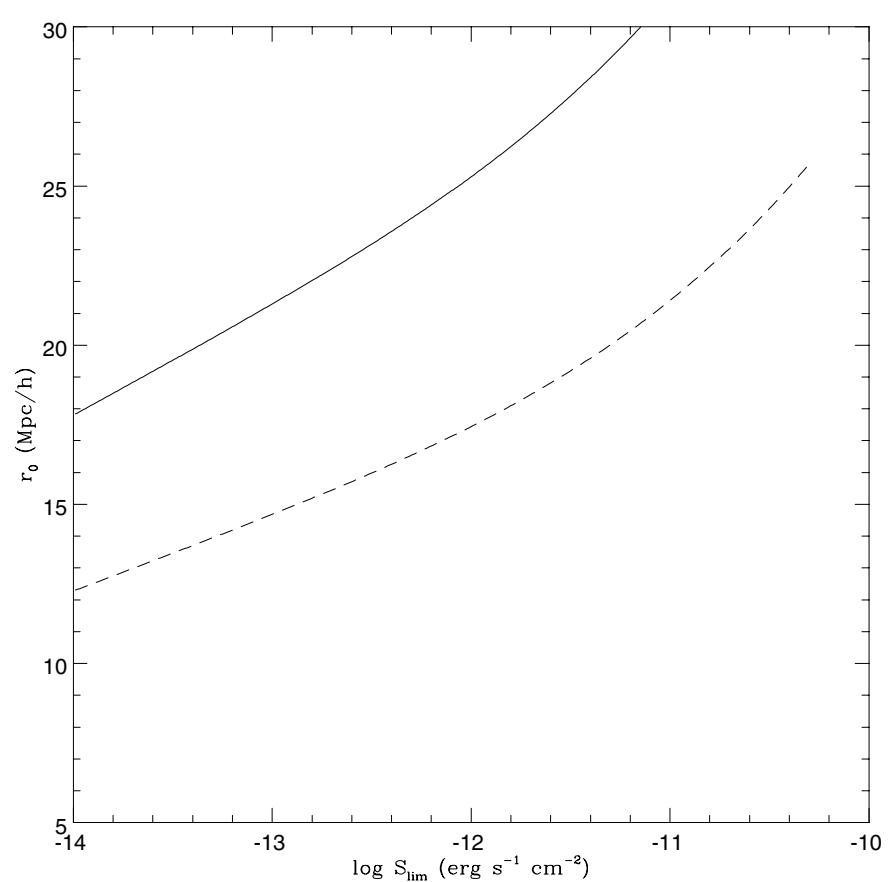

Fig. 6. The behavior of the correlation length $r_{0}$ as a function of the limiting X-ray flux $S_{\text {lim. }}$. The solid line represents the $\Lambda \mathrm{CDM}$ model according to the model of this paper, and the dashed line that of Moscardini et al. (2000a).

of this paper (differences in cosmological models parameters adopted), it is clear that there is a qualitative agreement but MMM tends to predict smaller correlation lengths (by approximately 20-30\%) with respect to S2000 and the latter predicts smaller correlation lengths than this paper. Part of the difference between MMM and S2000 comes from the different values of the exponent of the temperature used by the quoted authors in the temperature-luminosity relation: in $\mathrm{MMM} \mathcal{B}=1 / 3$ (see Sect. 2.4), and that used in S2000 ( $\alpha=1 / 3.4$ (see their Eq. (11))). Moreover, in their approach S2000 include a method to take account of redshift-space distortion effects not completely considered by $\mathrm{MMM}^{7}$ which tends to increase the correlation estimates.

In the present paper, the correlation lengths are larger in comparison with both the previous two quoted papers predictions. These differences, expecially when we compare the results with those of S2000, are due essentially to the different $M-T$ relation, mass function and bias model used in this paper. As reported in Sect. 2, the fundamental goal of this paper is to study the effects of the improvements quoted above on the values of the, cosmological parameters, and then to constrain the cosmological parameters by the clustering properties of clusters of galaxy using a maximum-likelihood analysis. This analysis was started by considering as a free parameter $\Omega_{\mathrm{m}}$ only, fixing $\Gamma=0.2$, which is in the range suggested by various other works (see e.g. Peacock \& Dodds 1996), and $\sigma_{8}$ to reproduce the cluster abundance.

For the normalization we adopt the fitting formula by Pierpaoli et al. (2003), with the $M-T$ normalization parameter $T_{*}=1.75$ (see Pierpaoli et al. 2003).

\footnotetext{
7 They used a zero-order model similar to that of Kaiser.
}

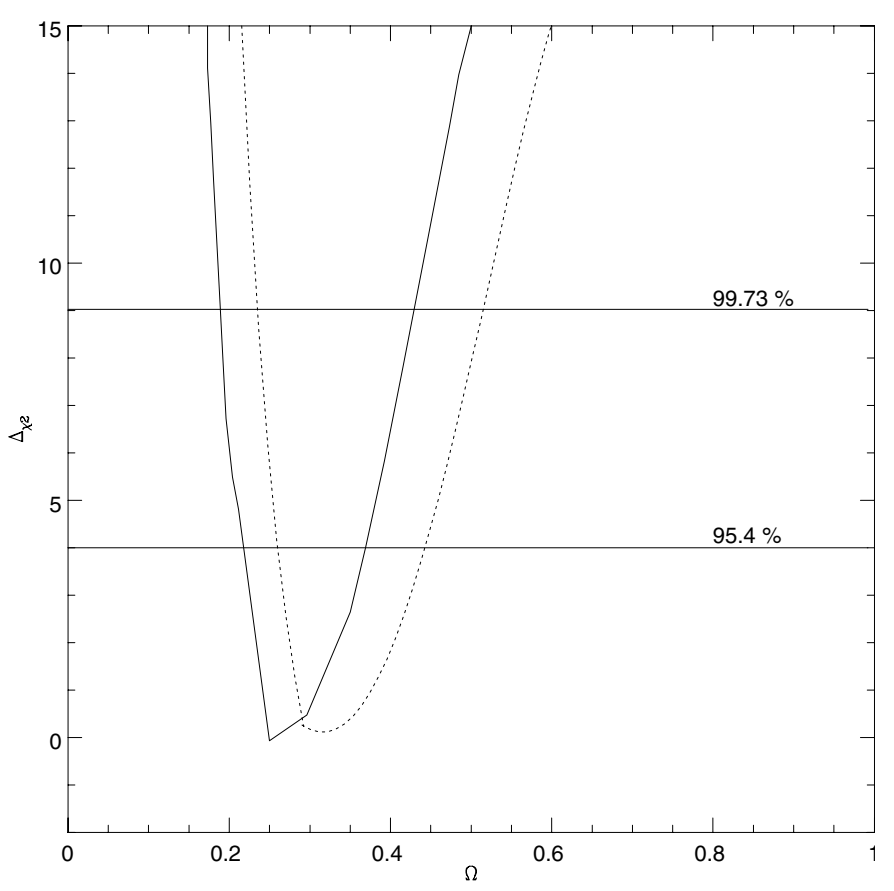

Fig. 7. The variation of $\Delta_{\chi^{2}}$ around the best-fitting value of the matter density parameter $\Omega_{0 \mathrm{~m}}$ for flat CDM models (with varying cosmological constant $\Omega_{0 \Lambda}=1-\Omega_{0 \mathrm{~m}}$ ) with shape parameter $\Gamma=0.2$ and normalization reproducing the cluster abundance. The solid line represents the result of MMM (obtained using the X-ray complete cluster dataset), and the dashed line that of this paper. Horizontal lines corresponding to the 95.4 and 99.73 per cent confidence levels are also shown.

In Fig. 7, we plot the results of the maximum likelihood analysis obtained by using X-ray band data (RASS1, BCS, XBACs, REFLEX) for flat models with varying cosmological constant $\left(\Omega_{0 \Lambda}=1-\Omega_{0 \mathrm{~m}}\right)$, with $\Gamma=0.2$. The solid line represents the results obtained using the complete X-ray data set by MMM, and the dotted line gives the results for the model in the present paper.

The constraints on $\Omega_{\mathrm{m}}$ are: $0.25 \leq \Omega_{0 \mathrm{~m}} \leq 0.45$ and $0.23 \leq$ $\Omega_{0 \mathrm{~m}} \leq 0.52$ at 95.4 and 99.73 per cent levels, respectively. The result shows larger values for $\Omega_{0 \mathrm{~m}}$ on the order of $20-30 \%$, when compared with MMM results $\left(0.2 \leq \Omega_{0 \mathrm{~m}} \leq 0.35\right.$ and $0.2 \leq \Omega_{0 \mathrm{~m}} \leq 0.45$ at the 95.4 and 99.73 per cent levels, respectively).

In Fig. 8 we show the results of the maximum likelihood analysis fixing the model normalization to reproduce the cluster abundance, and leaving two free parameters: $\Gamma$, and $\Omega_{\mathrm{m} 0}$. The solid lines represents the 99.73 confidence levels, and the dashed lines the 95.4 confidence levels. The figure shows that the allowed regions, at least for $\Omega_{0 \mathrm{~m}} \geq 0.5$, depend strongly on $\Gamma$. In the case of the flat model (Fig. 8a), the constraints for $\Gamma$ are $0.1 \leq \Gamma \leq 0.14$, and in the case of the open model (Fig. 8b) $0.09 \leq \Gamma \leq 0.13$. These values are smaller than those of MMM, by about 20-30\%. (Note that in MMM paper, the reported values are approximated. A comparison of our and their confidence contours shows the difference).

In Fig. 9 we show the constraints in the $\Gamma-\sigma_{8}$ plane (after keeping the values of $\Omega_{\Lambda}$ fixed). We consider an open model 


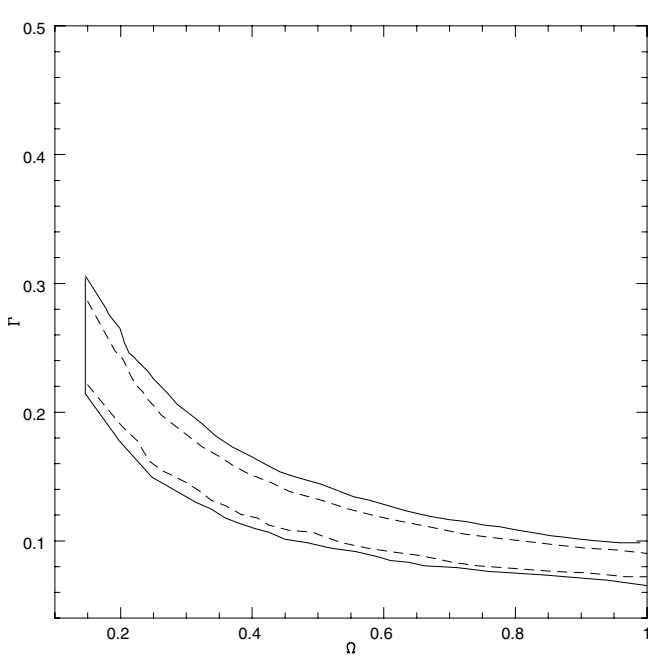

(a)

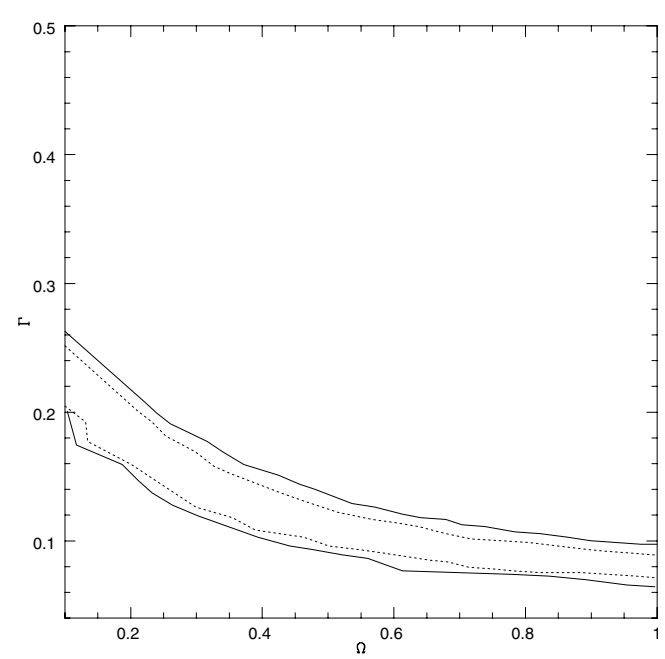

(b)

Fig. 8. Confidence contours of $\Gamma$ and $\Omega_{0 \mathrm{~m}}$. Dashed and solid lines represent 95.4 and 99.73 per cent confidence levels. The left panel refers to flat cosmological models with varying cosmological constant the right one to open models with vanishing $\Omega_{0 \Lambda}$.

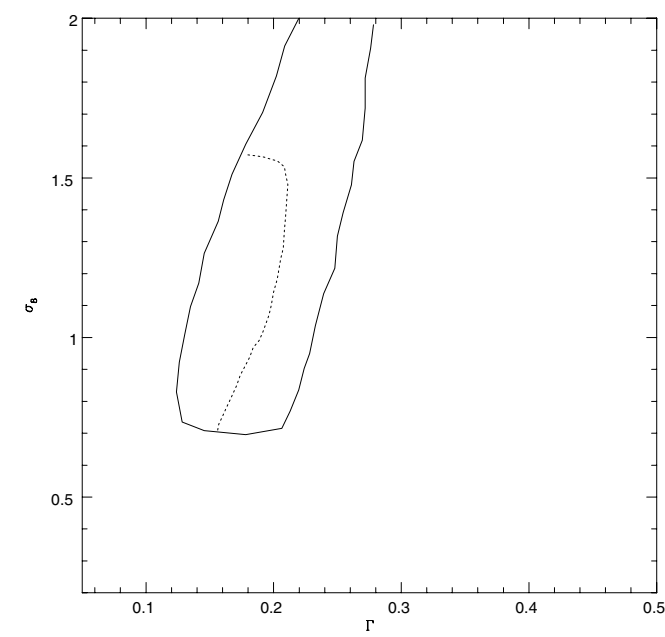

(a)

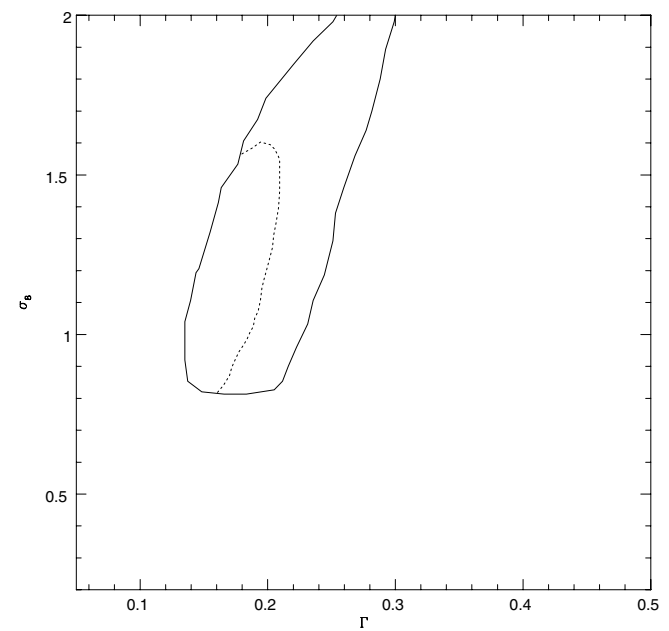

(b)

Fig. 9. Confidence contours of $\Gamma$ and $\sigma_{8}$. Dotted and solid lines represent 95.4 and 99.73 per cent confidence levels. Panel a) refers to a CDM model with $\Omega_{0 \mathrm{~m}}=0.3, \Omega_{0 \Lambda}=0$. Panel b) refers to a CDM model with $\Omega_{0 \mathrm{~m}}=0.3, \Omega_{0 \Lambda}=0.7$.

with $\Omega_{0 \mathrm{~m}}=0.3$, Fig. 9a, and a flat model, Fig. 9b, again with $\Omega_{0 \mathrm{~m}}=0.3^{8}$.

For the open model with $\Omega_{0 \mathrm{~m}}=0.3$ the $2 \sigma$ region has $\Gamma$ in the range $0.11-0.2$ and $\sigma_{8}$ between 0.7 and 1.55 . The values of $\sigma_{8}$ obtained here are larger by $\simeq 20 \%$ than those of MMM.

For a flat model with $\Omega_{0 \mathrm{~m}}=0.3$ the $2 \sigma$ region has $0.13 \leq$ $\Gamma \leq 0.2$ and $0.8 \leq \sigma_{8} \leq 1.6$. The values of $\sigma_{8}$ obtained are larger than those of MMM by $\simeq 20 \%$

The effect of varying $\alpha$ on the maximum-likelihood analysis has been also studied. From the study, it turns out that $\Gamma$ is quite insensitive to a change of $\alpha$, while $\sigma_{8}$ is only weakly dependent on it: the minimum in the maximum-likelihood analysis decreases (increases) by $\simeq 10 \%$ for $\alpha=2.5$, (for $\alpha=3.5$ ) (see also Fig. 8 of Borgani et al. 1999). For $\Omega_{0 \mathrm{~m}}$ the changes are

\footnotetext{
${ }^{8}$ Note that the value of $\Omega_{0 \mathrm{~m}}$ chosen is not too different from that obtained by WMAP: $\Omega_{0 \mathrm{~m}} h^{2}=0.14 \pm 0.02$ and $h=0.72 \pm 0.05$ (Spergel et al. 2003).
}

similar to those of $\Gamma$ but larger (of the order of $20 \%$ ) (see also Fig. 4 of Borgani et al. 2001; and Fig. 10 of Eke et al. 1998).

Notice that in the analysis of this paper, we used only the data from the X-ray catalogues and not a combination of optical and X-ray catalogues. This is because, as shown by MMM, the combination of optical and X-ray catalogues gives results that are almost indistinguishable from those obtained by the X-ray analysis only. As in MMM, the constraints from the Xray datasets are in general tighter than those obtained from the optical data.

Although the quoted uncertainties have so far been of minor importance because of the paucity of observational data, a breakthrough is needed in the quality of the theoretical modeling if high-redshift clusters are to take part in the highprecision-era of observational cosmology. As shown, using models like PS for the mass function instead of models that are in agreement with Jenkins et al. (2001), introduces errors 
of the order of $20-30 \%$ in the values of the parameters constrained (see Del Popolo 2003) and other errors are introduced if one uses a simplified version of the $M-T$ relation. Moreover a proper interpretation of such redshift surveys in terms of the clustering evolution requires an understanding of many cosmological effects which can be neglected for $z \ll 1$ and thus have not been considered seriously so far. For example, neglecting the light-cone effect leads to underestimates of $\simeq 20 \%$ in samples like RASS1, and of up to $25 \%$ for a deeper survey such ABRIXAS (Moscardini et al. 2000a). Neglecting red-shift space distortions produces underestimates of $r_{0}$ of $\simeq 10 \%$ : as reported in the present paper, a comparison between the behavior of the correlation length as a function of the limiting X-ray flux, in papers like S2000 and MMM shows a difference of $\simeq 30 \%$ because in MMM the redshift-space distortion effects were not taken into account, and because of a difference in the exponent in the temperature-luminosity relation. Taking into account the asphericity in gravitational collapse that leads to a different relation for bias (Del Popolo \& Gambera 1998; Sheth \& Tormen 1999), to a modified version of the mass function (Del Popolo \& Gambera 1998; Sheth \& Tormen 2002; Del Popolo 2002b), and a different $M-T$ relation (Del Popolo 2002a), leads to higher values of $\Omega_{0 \mathrm{~m}}, \sigma_{8}, r_{0}$ by at least $20 \%$. In the near future theory and observations should converge towards a more precise constraining of cosmological parameters.

\section{Discussion and conclusions}

In this paper, we have recalculated the two-point correlation function of clusters of galaxies for OCDM and $\Lambda$ CDM cosmological models, improving the model of S2000, in the light of recent theoretical developments, by using the theoretical mass function derived in Del Popolo (2002a), the $M-T$ relation derived in Del Popolo (2002b) and the bias model of Del Popolo (2001). As in Suto's paper, the model properly takes account of nonlinear gravitational evolution of mass fluctuations, redshift-space distortion due to the linear peculiar velocity field and to finger-of-god, cluster abundance and bias evolution, and the light-cone effect. This theoretical model has before been compared with the observed spatial correlation function for clusters in RASS1, and in XBACs samples. The comparison shows that only the predictions of models with $\Omega_{\mathrm{m}}=0.3$ are in good agreement with data. The results are in qualitative agreement with MMM, but the values for $r_{0}$ here obtained $\left(r_{0} \simeq 28.2 h^{-1} \mathrm{Mpc}\right.$ for the $\Lambda \mathrm{CDM}$ model) are larger than the values of the quoted paper (MMM), $\left(r_{0} \simeq\right.$ $22 h^{-1} \mathrm{Mpc}$ for $\left.\Lambda \mathrm{CDM}\right)$, while they are in better agreement with that of Borgani et al. (1999), who found a value of $r_{0}=$ $26_{-4.7}^{+4.1} h^{-1} \mathrm{Mpc}$. In order to study the possible dependence of the clustering properties of the X-ray clusters on the observational characteristics defining the survey, we plot the values of the correlation length $r_{0}$ in the catalogues where we vary the limiting X-ray flux $S_{\lim }{ }^{9}$. All the cosmological models displays an

\footnotetext{
9 Notice that this analysis can be related to the study of the richness dependence of the cluster correlation function. In fact, a change in the observational limits implies a change in the expected mean intercluster separation $d_{\mathrm{c}}$.
}

increase of $r_{0}$ with $L_{\mathrm{lim}}$. The increase is more rapid for $\Lambda \mathrm{CDM}$ models (see MMM). Comparing the result with those of a similar analysis by S2000, (see their Fig. 8) and MMM it is clear that there is a qualitative agreement but MMM tends to predict smaller correlation lengths (by approximately 20-30\%) with respect to $\mathrm{S} 2000$, and the latter predicts smaller correlation lengths than the present paper. These differences, expecially when we compare the results with those of S2000, are due essentially to the different $M-T$ relation and mass function used in this paper. In order to obtain constraints on cosmological parameters we performed a maximum-likelihood analysis by comparing the theoretical predictions to a set of observational data in the X-ray band (RASS1 Bright Sample, BCS, XBACs, REFLEX), similarly to MMM. The parameters to be constrained are: $\Omega_{\mathrm{m}}, \Omega_{\Lambda}$, the power-spectrum shape parameter $\Gamma$ and the normalization $\sigma_{8}$. The constraints obtained for the matter density parameter in a flat CDM model, are: $0.25 \leq \Omega_{0 \mathrm{~m}} \leq 0.45$ and $0.23 \leq \Omega_{0 \mathrm{~m}} \leq 0.52$ at the 95.4 and 99.73 per cent levels, respectively, larger by $\simeq 20 \%$ than the MMM predictions. Keeping the model normalization fixed to reproduce the cluster abundance, and leaving two free parameters: $\Gamma$, and $\Omega_{\mathrm{m} 0}$, we find that for the flat model the constraints for $\Gamma$ are $0.1 \leq \Gamma \leq 0.14$, while for the open model $0.09 \leq \Gamma \leq 0.13$. These values are smaller than those of MMM, by about $20-30 \%$. After fixing the values of $\Omega_{\Lambda}$, we obtain the constraints in the $\Gamma-\sigma_{8}$ plane, showing that if we keep the value of $\Omega_{\Lambda}$ fixed the open model with $\Omega_{0 \mathrm{~m}}=0.3$ the $2 \sigma$ region has $\Gamma$ in the range $0.11-0.2$ and $\sigma_{8}$ between 0.7 and 1.55 . In the flat model with $\Omega_{0 \mathrm{~m}}=0.3$ the $2 \sigma$ region has $0.13 \leq \Gamma \leq 0.2$ and $0.8 \leq \sigma_{8} \leq 1.6$ In all three cases, the values of $\sigma_{8}$ obtained are larger than those of MMM by $\simeq 20 \%$. Varying $\alpha$, it turns out that $\Gamma$ is quite insensitive to the change of $\alpha$, while $\sigma_{8}$ is only weakly dependent on it: the minimum in the maximumlikelihood analysis decreases (increases) by $\simeq 10 \%$ for $\alpha=2.5$, (for $\alpha=3.5$ ). In the case of $\Omega_{0 \mathrm{~m}}$ the changes are similar to those of $\Gamma$ but larger (of the order of $20 \%$ ). Allowing the shape parameter to vary, we find that the clustering properties of clusters are almost independent of the matter density parameter and of the presence of a cosmological constant, while they appear to be strongly dependent on the shape parameter. The constraints from X-ray data are tighter than those coming from optical data. In conclusion, the data on clustering properties of galaxies can be used to constrain important cosmological parameters like $\Omega_{0 \mathrm{~m}}, \Gamma$ and $\sigma_{8}$.

Acknowledgements. A. Del Popolo would like to thank Boğaziçi University Research Foundation for the financial support through the project code 01B304.

\section{References}

Abadi, M. G., Lambas, D. G., \& Muriel, H. 1998, ApJ, 507, 526

Afshordi, N., \& Cen, R. 2002, ApJ, 564, 669

Bahcall, N. A., \& Soneira, R. M. 1983, ApJ, 270, 20

Bahcall, N. A., \& Cen, R. 1992, ApJ, 398, L81

Bahcall, N. A., \& West, M. J. 1992, ApJ, 392, 419

Bahcall, N. A., \& Fan, X. 1998, ApJ, 504, 1

Bahcall, N. A., Fan, X., \& Cen, R. 1997, ApJ, 485, L53

Bardeen, J. M., Bond, J. R., Kaiser, N., \& Szalay, A. S. 1986, ApJ, 304,15 
Blanchard, A., \& Bartlett, J. G. 1997, A\&A, 332, L49

Blanchard, A., Bartlett, J. G., \& Sadat, R. 1998, in Les Comptes Rendus de l'Académie des Sciences

Borgani, S., Girardi, M., Carlberg, R. G., Yee, H. K. C., \& Ellingson E. 1999, ApJ, 527, 561

Borgani, S., Plionis, M., \& Kolokotronis, V. 1999, MNRAS, 305, 866

Borgani, S., Rosati, P., Tozzi, P., et al. 2001, ApJ, 561, 13

Böhringer, H., Guzzo, L., Collins, C. A., et al. 1998, Messenger, 94, 21

Bryan, G. L., \& Norman, M. L. 1998, ApJ, 495, 80

Catelan, P., Lucchin, F., Matarrese, S., \& Porciani, C. 1998, MNRAS, 297, 692

Cole, S., Fisher, K. B., \& Weinberg, D. H. 1994, MNRAS, 267, 785

David, L. P., Slyz, A., Jones, C., Forman, W., \& Vrtilek, S. D. 1993, ApJ, 412, 479

De Grandi, S., Böhringer, H., Guzzo, L., et al. 1999a, ApJ, 514, 148

De Grandi, S., Guzzo, L., Böhringer, H., et al. 1999b, ApJ, 513, L17

Del Popolo, A. 2001, MNRAS, 326, 667

Del Popolo, A. 2002a, MNRAS, 336, 81

Del Popolo, A. 2002b, MNRAS, 337, 529

Del Popolo, A. 2003, ApJ, 599, 723

Del Popolo, A., \& Gambera, M. 1998, A\&A, 337, 96

Del Popolo, A., \& Gambera, M. 1999, A\&A, 344, 17

Del Popolo, A., \& Gambera, M. 2000, A\&A, 357, 809

Del Popolo, A., Ercan, E. N., \& Xia, Z. Q. 2001, AJ, 122, 487

Donahue, M., \& Voit, G. M. 1999, ApJ, 523, 137

Donahue, M., Voit, G. M., Scharf, C. A., et al. 1999, ApJ, 527, 525

Ebeling, H., Voges, W., Böhringer, H., et al. 1996, MNRAS, 283, 1103

Ebeling, H., Edge, A. C., Böhringer, H., et al. 1998, MNRAS, 301, 881

Efstathiou, G., Dalton, G. B., Sutherland, W. J., \& Maddox, S. J. 1992, MNRAS, 257, 125

Eke, V. R., Cole, S., Frenk, C. S., \& Henry, J. P. 1998, MNRAS, 298, 1145

Fan, X., Bahcall, N. A., \& Cen, R. 1997, ApJ, 490, L123

Governato, F., Babul, A., Quinn, T., et al. 1999, MNRAS, 307, 949

Hamilton, A. J. S., Kumar, P., Lu, E., \& Mathews, A. 1991, ApJ, 374, L1

Hamana, T., Yoshida, N., Suto, Y., \& Evrard, A. 2001, ApJ, 561, 143

Hamilton, A. J. S. 1998, in The Evolving Universe. Selected Topics on Large-Scale Structure and on the Properties of Galaxies (Dordrecht: Kluwer), 185

Hauser, M. G., \& Peebles, P. J. E. 1973, ApJ, 185, 757

Henry, J. P. 2000, ApJ, 534, 565

Jenkins, A., Frenk, C. S., White, S. D. M., et al. 2001, MNRAS, 321, 372

Jing, Y. P. 1998, ApJ, 503, L9

Jing, Y. P. 1999, ApJ, 515, L45

Kaiser, N. 1987, MNRAS, 227, 1

Kravtsov, A. V., \& Klypin, A. A. 1999, ApJ, 520, 437

Kauffmann, G., Colberg, J. M., Diaferio, A., \& White, S. D. M. 1999, MNRAS, 303, 188

Klypin, A. A., \& Kopylov, A. I. 1983, Sov. Astr. Lett., 9, 41

Kitayama, T., \& Suto, Y. 1997, ApJ, 490, 557

Kitayama, T., Sasaki, S., \& Suto, Y. 1998, PASJ, 50, 1

Lacey, C., \& Cole, S. 1993, MNRAS, 262, 627

Lacey, C., \& Cole, S. 1994, MNRAS, 271, 676

Liddle, A. R., Lyth, D. H., Roberts, D., \& Viana, P. T. P. 1996a, MNRAS, 278, 644
Liddle, A. R., Lyth, D. H., Viana, P. T. P., \& White, M. 1996b, MNRAS, 282, 281

Magira, H., Jing, Y. P., \& Suto, Y. 2000, ApJ, 528, 30

Marshall, H. L., Avni, Y., Tananbaum, H., \& Zamorani, G. 1983, ApJ, 269, 35

Matarrese, S., Coles, P., Lucchin, F., \& Moscardini, L. 1997, MNRAS, 286, 115

Mathiesen, B., \& Evrard, A. E., 1998, MNRAS, 295, 769

Matsubara, T., \& Suto, Y. 1996, ApJ, 470, L1

Matsubara, T., Suto, Y., \& Szapudi, I. 1997, ApJ, 491, L1

Mo, H. J., \& White, S. D. M. 1996, MNRAS, 282, 347

Mo, H. J., Jing, Y. P., \& Börner, G. 1997, MNRAS, 286, 979 (MJB)

Moscardini, L., Coles, P., Lucchin, F., \& Matarrese, S. 1998, MNRAS, 299, 95

Moscardini, L., Matarrese, S., Lucchin, F., \& Rosati, P. 2000a, MNRAS, 316, 283

Moscardini, L., Matarrese, S., De Grandi, S., \& Lucchin, F. 2000b, MNRAS, 314, 647

Moscardini, L., Matarrese, S., \& Mo, H. J. 2001, MNRAS, 327, 422

Mushotzky, R. F., \& Scharf, C. A. 1997, ApJ, 482, L13

Nakamura, T. T., Matsubara, T., \& Suto, Y. 1998, ApJ, 494, 13

Nishioka, H., \& Yamamoto, K. 1999, ApJ, 520, 426

Peacock, J. A., \& Dodds, S. J. 1994, MNRAS, 267, 1020

Peacock J. A., \& Dodds, S. J. 1996, MNRAS, 280, L19

Pierpaoli, E., Borgani, S., Scott, D., \& White, M. 2003, MNRAS, 342, 163

Ponman, T. J., Bourner, P. D. J., Ebeling, H., \& Böhringer, H. 1996, MNRAS, 283, 690

Porciani, C., Catelan, P., \& Lacey, C. 1999, ApJ, 513, L99

Postman, M. 1999, Evolution of large scale structure: from recombination to Garching, ed. A. J. Banday, R. K. Sheth, \& L. N. da Costa, Garching, Germany: European Southern Observatory [1999], Proceedings of the MPA-ESO cosmology Conf., Garching, Germany, 2-7 August 1998, 270

Press, W. H., \& Schechter, P. 1974, ApJ, 187, 425

Reichart, D. E., Castander, F. J., \& Nichol, R. C. 1999a, ApJ, 516, 1

Reichart, D. E., Nichol, R. C., Castander, F. J., et al. 1999b, ApJ, 518, 521

Sadat, R., Blanchard, A., \& Oukbir, J. 1998, A\&A, 329, 21

Sheth, R. K., \& Tormen, G. 1999, MNRAS, 308, 119

Sheth, R. K., \& Lemson, G. 1999, MNRAS, 304, 767

Sheth, R. K., \& Tormen, G. 2002, MNRAS, 329, 61 (ST)

Sheth, R. K., Mo, H. J., \& Tormen, G. 2001, MNRAS, 323, 1 (SMT)

Smith, R. E., Peacock, J. A., Jenkins, A., et al. 2003, MNRAS, 341, 1311

Spergel, D. N., Verde, L., Peiris, H. V., et al. 2003, ApJS, 148, 175

Sugiyama, N. 1995, ApJS, 100, 281

Suto, Y., Yamamoto, K., Kitayama, T., \& Jing, Y. P. 2000, ApJ, 534, 551

Taruya, A., \& Suto, Y. 2000, ApJ, 542, 559

Viana, P. T. P., \& Liddle, A. R. 1999, MNRAS, 303, 535

Voit, C. M., \& Donahue, M. 1998, ApJ, 500, 111

Voit, C. M. 2000, ApJ, 543, 113

White, S. D. M., Jones, C., \& Forman, W. 1997, MNRAS, 292, 419

Wu, X. P., Xue, Y. J., \& Fang, L. Z. 1999, ApJ, 524, 22

Yamamoto, K., \& Suto, Y. 1999, ApJ, 517, 1

Yamamoto, K., Nishioka, H., \& Suto, Y. 1999, ApJ, 527

Yoshikawa, K., Taruya, A., Jing, Y. P., \& Suto, Y. 2001, ApJ, 558, 520 